\title{
Difficulties in Recruitment for a Randomised Controlled Trial of Lifestyle Intervention for Type 2 Diabetes: Implications for Diabetes Management
}

\author{
George A. Jelinek ${ }^{1,2 *}$, Emily Hadgkiss ${ }^{1}$, Craig Hassed ${ }^{3}$, Bernard Crimmins ${ }^{4}$, Peter Schattner ${ }^{5}$, \\ Danny Liew ${ }^{6}$, Rick Kausman ${ }^{7}$, Warrick J. Inder ${ }^{8}$, Siegfried Gutbrod ${ }^{9}$, Tracey J. Weiland ${ }^{1,2}$ \\ ${ }^{1}$ Emergency Practice Innovation Centre, St. Vincent's Hospital, Melbourne, Australia \\ ${ }^{2}$ Department of Medicine, The University of Melbourne, St. Vincent's Hospital, Melbourne, Australia \\ ${ }^{3}$ Department of General Practice, Faculty of Medicine, Nursing and Health Sciences, Monash University, Clayton, Australia \\ ${ }^{4}$ Manningham General Practice, Melbourne, Australia \\ ${ }^{5}$ East Bentleigh General Practice, Melbourne, Australia \\ ${ }^{6}$ Department of Medicine, Faculty of Medicine and Health Sciences, The University of Melbourne, Royal Melbourne Hospital, \\ Melbourne, Australia \\ ${ }^{7}$ The Butterfly Foundation, Malvern, Australia \\ ${ }^{8}$ Formerly Department of Endocrinology, St. Vincent's Hospital, Melbourne, Australia \\ ${ }^{9}$ The Gawler Foundation, Yarra Junction, Australia \\ Email: *George.jelinek@svhm.org.au
}

Received July 23, 2012; revised August 25, 2012; accepted September 27, 2012

\begin{abstract}
Objective: To report our experience of attempting a randomised controlled trial of an intensive lifestyle intervention for early type 2 diabetes delivered in a residential setting. Methods: We established a trial requiring 84 participants (46 standard care and 38 intervention) to detect a 1\% difference in HbA1c between intervention and control groups at 12 months, allowing for attrition. Ethics approval was obtained from Monash University. Results: The study was abandoned after five months of consistent promotion due to recruitment failure (four subjects recruited). Conclusion: It appears to be difficult for patients with diabetes to commit to a live-in period of education regarding lifestyle modification as a means of treating the illness. We recommend better education of patients and their doctors about the potential health benefits of lifestyle change to manage type 2 diabetes, and further research into novel methods of delivering lifestyle advice which are both effective and sustainable.
\end{abstract}

Keywords: Type 2 Diabetes Mellitus; Randomised Controlled Trial; Management; Lifestyle; Risk Factors

\section{Introduction}

Type 2 diabetes mellitus (T2DM) is a global epidemic. The landmark Australian Diabetes Obesity and Lifestyle (AusDiab) study found that Australia has one of the highest T2DM prevalences in the developed world [1], with $7.4 \%$ of the population affected [1]. A quarter of all adult Australians have either diabetes or pre-diabetes [1] with 275 adults developing T2DM every day [2]. In Australia, $50 \%$ of diabetics remain undiagnosed, and are thus at risk of developing severe complications that could increase morbidity and mortality [3] and hence healthcare costs. The magnitude of the rapidly rising prevalence rates of T2DM is compounded by the high prevalence of risk factors for T2DM, suggesting that the incidence rate of diabetes will increase substantially in the

*Corresponding author. future. The severe consequences of T2DM impose a heavy burden on patients and society in terms of patient outcomes and financial costs. Diabetes is the eighth leading cause of death worldwide [4]. With regard to finances, diabetes-attributable costs in Australia amount to $\$ 7$ billion annually, with complications the major driver of these costs [5]. Left untreated, T2DM can cause severe complications [6] which increase mortality [7] and double healthcare costs [5]. Symptoms of T2DM often do not appear until seven to twelve years after the true onset of T2DM [8]. By the time of diagnosis, up to 50\% of newly diagnosed diabetics have developed diabetic complications [3].

Type 2 diabetes is often described as a lifestyle disease. This is because the disease and its complications may be the result of lifestyle factors such as poor diet, obesity and insufficient exercise. Consistent with this there has 
been a significant emphasis on prevention of diabetes through lifestyle interventions, particularly those that focus on diet and exercise. The major diabetes prevention trials indicate that lifestyle interventions have major benefits that are similar in different ethnic groups, and that they can halve the risk of T2DM in those at high-risk [9]. Recent systematic reviews have demonstrated lifestyle interventions (diet and exercise) to be at least as effective as pharmacological interventions in reducing the rate of progression to T2DM in people with impaired glucose tolerance $[10,11]$. Lifestyle interventions are becoming increasingly recognized as effective strategies to improve metabolic control [12] and protect against complications of T2DM such as microvascular disease [13]. Although under-utilized, lifestyle interventions are a key treatment component to managing established T2DM. Several studies have shown benefits from such interventions [14-19].

Given the burden associated with T2DM and its complications, the associated costs faced by the community, and the success of lifestyle interventions in preventing T2DM, we felt it important to assess whether comprehensive sustained lifestyle interventions could lessen the impact of established diabetes, and the extent to which reversion to normal glucose control is possible. Clearly attaining long term genuine health with improved diet, weight loss, regular exercise and normal glycaemic control is an important objective if it is both deliverable and sustainable in a community setting. Recent trials incurporating lifestyle interventions have called for an increase in intensity of the intervention. Previous studies have examined lifestyle interventions delivered either on an individual basis or in a non-residential group setting over several sessions. While studies spanning several years have been conducted, few provide follow-up extending beyond the cessation of the intervention. To our knowledge no studies have examined the effects of lifestyle intervention against standard care delivered in an intensive, residential "live-in" setting.

We have previously examined the feasibility of screening for type 2 diabetes in emergency departments (EDs) [20], where one might expect a high prevalence of the disease. The study was limited by a very low follow up rate for formal testing with oral glucose tolerance tests. In pursuing explanations, we examined patient and emergency clinician attitudes to T2DM and prediabetes [21], demonstrating that while clinicians were more aware of prediabetes than patients, both groups were largely unaware of the degree to which prediabetes and T2DM can be modified or controlled by lifestyle changes.

While the literature clearly indicates the potential for lifestyle change to improve the course of T2DM [10,11], data on concerted lifestyle change as a method for controlling T2DM has been relatively sparse. We sought to address this by conducting a randomised controlled trial of an intensive live-in week-long retreat promoting lifestyle change versus standard care, with the aim of also examining the duration of the effect beyond the period of intervention. Here we report the methodology of the study, our difficulties in recruitment necessitating abandonment of the study, and the implications of this lack of engagement by people with T2DM for the diabetes epidemic.

\section{Methodology}

We aimed to determine the feasibility and efficacy of a comprehensive lifestyle intervention delivered in a residential setting plus standard care compared to standard care alone in patients with recent onset T2DM. The primary outcome measure was to be difference in glycaemic control as assessed by fasting glucose and HbA1c.

We designed an open-label, randomized controlled trial, set in semi-rural Victoria, Australia. We aimed to include participants with T2DM aged 18 years or more, with newly identified ( $<24$ months) T2DM, able to attend a 1-week residential program and four 3-hour booster sessions over the subsequent 14 weeks. Importantly, they had to be willing to undertake significant lifestyle change. There was no cost involved; program fees were funded by the study. We excluded patients who were unable to provide informed consent or communicate in English, cognitively impaired or in an altered conscious state, receiving palliative care, and who had commenced sulfonylureas as an adjunct to metformin as it was felt that this could potentially precipitate hypoglycaemia in the study setting.

Patients were to be recruited from the ED and endocrinology clinic at St Vincent's Hospital Melbourne (SVHM), and external primary and community health centres throughout Melbourne. Many health professionals from these sites, including credentialed Diabetes Educators listed in Victoria with the Australian Diabetes Educators Association, were informed of the study, provided with advertising materials and instructions on how to refer patients. The study was advertised on several websites, including Diabetes Australia and Diabetes Victoria, on posters and brochures in various clinics, and by email to several healthcare and educational institutions. Patients were to be recruited over a 9-month period.

The treatment group, receiving lifestyle intervention (LI) plus standard therapy was to have intensive multimodal lifestyle intervention in a week-long residential program with daily group sessions educating about diabetes pathophysiology, risks, stress management, and lifestyle interventions such as diet, weight loss and exercise. Participants were to receive counseling and group interaction designed to improve compliance with lifestyle change. Briefly, the changes suggested were a low saturated fat plant-based wholefood diet, omega 3 suppli- 
mentation, vitamin D supplementation, exercise, and stress reduction. A group exercise program was devised by the researchers. Practical exercise sessions were to be accompanied by educational sessions.

For those randomized to LI arm, a series of four booster sessions was to be provided of 3 hours each across 14 weeks after the intervention, to consolidate knowledge and maintain motivation and support. At the completion of the intervention, those randomized to LI were to be referred to their usual medical officer or hospital clinic for continued management. In addition to receiving lifestyle intervention, participants were to receive standard care from their usual medical officer or hospital clinic. The standard therapy group was to continue to attend or be referred to their usual medical officer or hospital clinic as per usual practice.

We sought to measure several variables at baseline for both groups including: demographics, physiological indicators including glycaemic control (HbA1c), fasting glucose, lipid profile, vitamin $\mathrm{D}$, a number of atherosclerotic and inflammatory factors, body mass index, waist circumference, blood pressure, number of co-morbidities, diabetes symptoms, diet, exercise, smoking, alcohol intake, meditation, health service use, hypoglycaemic medications and psychosocial indicators includeing diabetes specific health-related quality of life and the Kessler Psychological Distress Scale. Participants of both groups were to be requested to attend SVHM for faceto-face follow-up at 12 months post-baseline, with reassessment of the measures, questionnaires and pathology recorded at baseline, with an additional tool assessing adherence in the LI group.

The primary outcomes were glycaemic control (HbA1c), and the percentage of patients achieving glycaemic control using fasting glucose (WHO definitions) and HbA1c (HbA1c < 7\%). Secondary outcomes were proportion of the groups able to maintain adequate glycaemic control (HbA1c < 7\%) without drugs, and number and dose of hypoglycaemic drugs prescribed. Other secondary outcomes were changes in those factors recorded at baseline.

The primary outcome measure was HbA1c at 12 months. A difference between group means of $1.0 \%$ HbA1c (e.g., 8.5 Vs 7.5) was taken as clinically meaningful [22]. With alpha at 0.05 (two tailed) and power at $80 \%$, a total of 64 participants were required, based on equal variance and standard deviation of 1.4 consistent with previous research $[17,19]$. We anticipated an attrition rate of $30 \%$ in the standard care group, and $15 \%$ in the intervention group. Therefore, we needed to recruit 84 participants (46 standard care and 38 intervention). Data were to be analyzed on an intention-to-treat basis using the "complete case" method with sensitivity analysis around assumptions for missing values.

\section{Results}

The study was approved by the Ethics Committee of Monash University on May 11, 2011, and the retreat scheduled for March 29, 2012. Advertising began in June, 2011. Despite concerted efforts at recruitment, only four participants were consented by the researchers, with two allocated to each of the arms. The study was formally abandoned on December 1, 2011, due to inability to recruit.

\section{Implications}

Our failure to recruit has significant implications for the growing problem of how to tackle the diabetes epidemic that threatens to overwhelm our health system. As with other chronic diseases, the most cost-effective approach is through prevention. Evidence-based strategies that engage patients to make lifestyle choices that result in better glucose control are urgently needed. We know that better glucose control has the potential to significantly decrease complications [23] and health care costs [5], and halt or reverse disease progression [24]. Our study sought to empower patients to make these lifestyle modifications, but despite the large number of eligible people in our population, we were frankly unable to engage them in this project. Patients may have been unwilling to participate in the program, given work, family or other commitments. Yet a similar five day live-in program, conducted for people with multiple sclerosis three times annually, at a cost, has a waiting list, and participants gain significant improvements in quality of life, and measures of physical and mental health [25,26]. This suggests that there is a relative lack of concern or fear associated with T2DM, perhaps due to the high incidence and insidious nature; perhaps, in an effort not to stigmatize or frighten people with the illness, having T2DM has been normalized in our community. We also had more interest from patients with diabetes of longer duration than two years. Perhaps those with early diabetes had rather less concern about the long-term health consequences of the illness when they were essentially well.

Our previous research suggests that people with T2DM do not understand the clear association of this illness with lifestyle, nor the therapeutic potential of lifestyle change [21]. Further, it seems likely from this research that doctors are also largely unaware of this potential, and may not be offering this option to their patients. The high prevalence of the disease may also be leading to a certain complacency on behalf of treating doctors. We noticed in our screening study [20] that patients were unlikely to attend for follow up after screening positive for T2DM, reasons among which were being advised by their doctors against having a formal oral glucose tolerance test because the screening result was “just a number”. This was despite national guidelines 
suggesting all at-risk patients be screened and counseled [27]. There may also be a certain complacency among patients; the opposite side of the coin to complacency is distress, frequently a positive motivator for change. Many people with a diagnosis of diabetes are initially asymptomatic, therefore this does not lead to the distress that for instance an MS diagnosis does and hence the lack of drive to make change.

It may not be appropriate however to conclude that the poor recruitment was due solely to poor patient or medical practitioner appreciation of the importance of diabetes. Study design may have played a role. The proposed program was very intensive and required a major time commitment by participants. It involved a week-long residential program, followed by four booster sessions of 3 hours each. This would appear to be more than many people with diabetes can commit to. While we distributed advertisements for the study widely and via the internet, we could have advertised in more locations such as daily newspapers. It is possible that other factors such as the inconvenience of having blood tests or difficulty accessing sample collection locations may also have played a part. Further, recruitment was limited to patients within the first 2 years of diagnosis of diabetes. These were system factors mitigating against good recruitment to the study. Similar larger lifestyle intervention programs for prevention of T2DM (the US Diabetes Prevention Program [28]) recruited over 3000 patients at risk of diabetes from 27 centres over very long periods for recruitment. While we used large general practices, diabetes websites and diabetes education nurses to assist recruitment, we may have had more success using more than one hospital diabetes clinic and emergency department.

Our study however highlights the difficulties of undertaking lifestyle research in type 2 diabetes compared with pharmacological research, for which there are often incentives for both doctors and patients to engage. The solution to the problem must be addressed through a shift in social and cultural attitudes around lifestyle change and a willingness to take action, by patient and doctor alike. We recommend better education of health professionals and patients about the potential benefits of lifestyle change in managing T2DM, and continued research into better and novel methods of delivering the relevant lifestyle education to patients. In particular, future studies should perhaps consider less intensive interventions, and target ways of sustaining the lifestyle improvements beyond the period of initial intervention.

\section{REFERENCES}

[1] D. Dunstan, S. Colagiuri, P. Zimmet, et al., "The Rising Prevalence of Diabetes and Impaired Glucose Tolerance: The Australian Diabetes, Obesity and Lifestyle Study," Diabetes Care, Vol. 25, No. 5, 2002, pp. 829-834. doi:10.2337/diacare.25.5.829
[2] E. L. M. Barr, D. J. Magliano, P. Z. Zimmet, et al., “The Australian Diabetes, Obesity and Lifestyle Study. Tracking the Accelerating Epidemic: Its Causes and Outcomes," International Diabetes Institute, Melbourne, 2006.

[3] S. F. Wetterhall, D. R. Olson, F. DeStefano, et al., "Trends in Diabetes and Diabetic Complications, 19801987,” Diabetes Care, Vol. 15, No. 8, 1992, pp. 960-967. doi:10.2337/diacare.15.8.960

[4] A. C. Powers, "Diabetes Mellitus. Harrison’s Principles of Internal Medicine,” The McGraw-Hill Companies, Inc., Ohio, 2005.

[5] S. Colagiuri, R. Colagiuri, B. Conway, et al., "DiabCo\$t Australia: Assessing the Burden of Type 2 Diabetes in Australia,” Diabetes Australia, Canberra, 2003.

[6] D. L. Kasper, E. Braunwald, A. S. Fauci, et al., "Harrison's Principles of Internal Medicine,” 16th Edition, The McGraw-Hill Companies, Inc., New York, 2005.

[7] P. Zimmet, K. G. M. M. Alberti and J. Shaw, “Global and Societal Implications of the Diabetes Epidemic," Nature, Vol. 414, No. 6865, 2001, pp. 782-787. doi:10.1038/414782a

[8] Australian Centre for Diabetes Strategies, "National Evidence Based Guidelines for the Management of Type 2 Diabetes Mellitus. Prince of Wales Hospital,” National Health and Medical Research Council, Sydney, 2005.

[9] J. Tuomilehto and J. Lindstrom, "The Principal Trials for Prevention of Type 2 Diabetes Have Now Confirmed That Type 2 Diabetes Can Be Prevented by Life Style Changes. Prevention of Type 2 Diabetes by Life Style," Journees Annuelles de Diabetologie de Hotel-Dieu, 2003, pp. 77-89.

[10] C. L. Gillies, K. R. Abrams, P. C. Lambert, et al., "Pharmacological and Lifestyle Interventions to Prevent or Delay Type 2 Diabetes in People with Impaired Glucose Tolerance: Systematic Review and Meta-Analysis,” (BMJ) British Medical Journal, Vol. 334, No. 7588, 2007, pp. 334-299. doi:10.1136/bmj.39063.689375.55

[11] A. Yuen, Y. Sugeng, T. J. Weiland, et al., "Lifestyle and Medication Interventions for the Prevention and Delay of Type 2 Diabetes in Pre-Diabetes: A Systematic Review of Randomised Controlled Trials," Australian and New Zealand Journal of Public Health, Vol. 34, No. 2, 2010, pp. 172-178.

[12] S. L. Norris, M. M. Engelgau and K. M. Narayan, "Effectiveness of Self-Management Training in Type 2 Diabetes: A Systematic Review of Randomized Controlled Trials," Diabetes Care, Vol. 24, No. 3, 2001, pp. 561-587. doi:10.2337/diacare.24.3.561

[13] P. Gaede, P. Vedel, H. H. Parving, et al., "Intensified Multifactorial Intervention in Patients with Type 2 Diabetes Mellitus and Microalbuminuria: The Steno Type 2 Randomised Study,” Lancet, Vol. 353, No. 9153, 1999, pp. 617-622. doi:10.1016/S0140-6736(98)07368-1

[14] J. S. Cramer, R. F. Sibley, D. P. Bartlett, et al., “An Adaptation of the Diabetes Prevention Program for Use with High-Risk, Minority Patients with Type 2 Diabetes,” Diabetes Education, Vol. 33, No. 3, 2007, pp. 503-508. 


\section{doi:10.1177/0145721707301680}

[15] M. J. Davies, S. Heller, T. C. Skinner, et al., "Effectiveness of the Diabetes Education and Self Management for Ongoing and Newly Diagnosed (DESMOND) Programme for People with Newly Diagnosed Type 2 Diabetes: Cluster Randomised Controlled Trial,” (BMJ) British Medical Journal, Vol. 336, 2008, pp. 491-495. doi:10.1136/bmj.39474.922025.BE

[16] T. A. Deakin, J. E. Cade, R. Williams, et al., "Structured Patient Education: The Diabetes X-PERT Programme Makes a Difference,” Diabetic Medicine, Vol. 23, No. 9, 2006, pp. 944-954. doi:10.1111/j.1464-5491.2006.01906.X

[17] S. H. Kim, S. J. Lee, E. S. Kang, et al., "Effects of Lifestyle Modification on Metabolic Parameters and Carotid Intima-Media Thickness in Patients with Type 2 Diabetes Mellitus,” Metabolism, Vol. 55, No. 8, 2006, pp. 10531059. doi:10.1016/j.metabol.2006.03.017

[18] C. K. Roberts and R. J. Barnard, "Effects of Exercise and Diet on Chronic Disease," Journal of Applied Physiology, Vol. 98, No. 1, 2005, pp. 3-30. doi:10.1152/japplphysiol.00852.2004

[19] M. Trento, P. Passera, M. Bajardi, et al., "Lifestyle Intervention by Group Care Prevents Deterioration of Type II Diabetes: A 4-Year Randomized Controlled Clinical Trial,” Diabetologia, Vol. 45, No. 9, 2001, pp. 1231-1239. doi:10.1007/s00125-002-0904-8

[20] G. A. Jelinek, T. J. Weiland, G. Moore, et al., "Screening for Type 2 Diabetes with Random Finger-Prick Glucose and Bedside HbA1c in an Australian Emergency Department," Emergency Medicine \& Australasian, Vol. 22, No. 5, 2010, pp. 427-434. doi:10.1111/j.1742-6723.2010.01333.X

[21] T. J. Weiland, M. Nguyen and G. A. Jelinek, "Illness Perception and Knowledge with Regard to Prediabetes and Type 2 Diabetes: A Pilot Study of Emergency Department Patients and Staff," European Journal of Emer- gency Medicine, Vol. 19, No. 6, 2011, pp. 353-358.

[22] Training in Flexible, Intensive Insulin Management to Enable Dietary Freedom in People with Type 1 Diabetes: Dose Adjustment for Normal Eating (DAFNE) Randomised Controlled Trial," (BMJ) British Medical Journal, Vol. 325, 2002, p. 746. doi:10.1136/bmj.325.7367.746

[23] Y. Ohkubo, H. Kishikawa, E. Araki, et al., "Intensive Insulin Therapy Prevents the Progression of Diabetic Microvascular Complications in Japanese Patients with Non-Insulin-Dependent Diabetes Mellitus: A Randomized Prospective 6-Year Study,” Diabetes Research and Clinical Practice, Vol. 28, No. 2, 1995, pp. 103-117. doi:10.1016/0168-8227(95)01064-K

[24] J. P. Burke, S. M. Haffner, S. P. Gaskill, et al., "Reversion from Type 2 Diabetes to Nondiabetic Status. Influence of the 1997 American Diabetes Association Criteria,” Diabetes Care, Vol. 21, No. 8, 1998, pp. 1266-1270. doi:10.2337/diacare.21.8.1266

[25] E. Hadgkiss, G. Jelinek, T. Weiland, et al., "Health-Related Quality of Life Outcomes at 1 and 5 Years after a Residential Retreat Promoting Lifestyle Modification for People with Multiple Sclerosis,” Neurological Sciences, Vol. 34, No. 2, 2012, pp. 187-195.

[26] M. P. Li, G. A. Jelinek, T. J. Weiland, et al., "Effect of a Residential Retreat Promoting Lifestyle Modifications on Health-Related Quality of Life in People with Multiple Sclerosis,” Quality in Primary Care, Vol. 18, No. 8, 2010, pp. 379-389.

[27] S. Colagiuri, D. Davies, S. Girgis, et al., "National Evidence Based Guideline for Case Detection and Diagnosis of Type 2 Diabetes,” Diabetes Australia, Canberra, 2009.

[28] W. C. Knowler, E. Barrett-Connor, S. E. Fowler, et al., "Reduction in the Incidence of Type 2 Diabetes with Lifestyle Intervention or Metformin," New England Journal of Medicine, Vol. 346, No. 6, 2002, pp. 393-403. doi:10.1056/NEJMoa012512 